\title{
RESEARCH ON INFORMATION REQUIREMENT OF FIRST-ORDER UNIVERSAL IMPLICATION OPERATORS IN FUZZY REASONING
}

Fu Lihua and He Huacan

Department of Computer Science \& Engineering, Northwestern Polytechnical University, $X i$ 'an 7l0072, P.R. China

Abstract: $\quad$ Based on the definition of linear specificity measure, this paper discusses detailedly the conditions on which the first-order universal implication operators satisfy the information boundedness principle in fuzzy reasoning, and gets the corresponding conclusion: when fuzzy propositions have positive measuring errors for their membership grades, first-order universal implication operators satisfy the information boundedness principle only if they are rejecting or restraining correlative; when they have negative ones, the operators satisfy the principle only if they are restraining correlative. This conclusion has important directive meaning for how to give the value of the general correlative coefficient $h$ in practical control application.

Key words: Universal logic, First-order universal implication, General correlation, General self-correlation, Measure of specificity, Information boundedness principle

\section{INTRODUCTION}

As is well known, fuzzy reasoning now has become a theoretical basis and an important method for the design and analysis of fuzzy controller. However, an important problem involved in fuzzy reasoning is how to define the implication operator. People have presented many different definitions ${ }^{[1-}$ 5], but the majority usually relies on the subjective experience without theoretical guide and analyses of their effectiveness, which are often given at will and blindly. In 1996, Universal Logic proposed by Prof. He provided a 
valid path to resolve this problem ${ }^{[10-12]}$. Universal implication is the implication connective of Universal Logic, which is a cluster of $R$ implications determined by the general correlation coefficient $h$ between propositions. In practical application, according to the inherent correlation between propositions, we can take a corresponding implication operator from the cluster, so that overcome effectively the blindness.

In fuzzy reasoning, the starting objects and the resulting object are all fuzzy sets, and the semantic interpretation associated with the output of the FMP process depends on the value of the resulting fuzzy subset at all points in its domain. Thus, in considering the appropriateness of the implication operator used in $F M P$, account must be taken of the whole resulting fuzzy subset, not only some points in its domain ${ }^{[7-9]}$. So, Yager proposed the global requirements for implication operators in fuzzy reasoning, called as information boundedness principle ${ }^{[8]}$.

In practical control application, how to determine the value of $h$ between propositions has been a problem for further studies. When taking no account of the measuring errors for the membership grades of propositions, we have proceeded the detailed research on information requirement of zero-order universal implication operators, and get an important conclusion ${ }^{[17]}$. However, in practical application, the measuring errors are ineluctable. Therefore, based on the information boundedness principle, this paper discusses further the information requirement of first-order universal implication operators in a specific way, and draws the general conclusion, which has important directive meaning for how to give the value of $h$.

\section{FUNDAMENTAL CONCEPTS}

\subsection{Basic model of fuzzy reasoning}

In fuzzy reasoning, the basic model of FMP can be represented as follows:

rule

$$
A \rightarrow B
$$

for given

$A^{*}$

to determine

$$
B^{*}
$$

where $A$ and $A^{*}$ are the fuzzy sets in $X$, and $B$ and $B^{*}$ are the fuzzy sets in $Y$.

\subsection{Basic idea of Universal Logic ${ }^{[10-12]}$}

1. Basic idea of Universal Logic 
Based on the investigation on the general regulation of the logics having existed, in 1996, Prof. He proposed firstly a kind of totally new theoretical frame of mathematical logic, called as Universal Logic. It is a continuous and parameterized logic system and describes the logic regulation of the flexible world. And it contains every kind of logics and reasoning forms. Its basic idea can be summarized as follows: it is the general correlation between propositions that makes logic operators are uncertain.

He leads the Chinese classical philosophic thought into the theory of flexible logics, which is all things in the world are correlative, namely either mutual promoting or restraining. So he uses the general correlation to explain why the logic operators of proposition connectives are not unique. The general correlation is the inherent character of things and contains general correlation and general self-correlation, which represent the correlation between fuzzy propositions and the measuring errors of the membership grades of fuzzy proposition, respectively.

And he uses general correlation coefficient $h$ to describe general correlation, changing continuously from 1 to 0 . General correlation changes continuously from the max-attracting state to the max-restraining state. As the tolerance decreasing from its maximum, general correlation decreases continuously from its max-attracting state $(h=1)$ to the independent correlation $\operatorname{state}(h=0.75)$, and then to the max-rejecting state(namely the min-restraining state, $h=0.5$ ). After that, with the restraint increasing, it continues decreasing from its min-restraining state $(h=0.5)$ to the maxrestraining state $(h=0)$ through its deadlock one $(h=0.25)$. Similarly, general self-correlation coefficient $k$ is used to describe general self-correlation, changing continuously from 0 to 1 , too. For example, if measuring errors are the positive maximums, then $k=1$, if they are the negative ones, then $k=0$, and if there are no measuring errors, then $k=0.5$. Thus, based on the theory of norms and the correlation coefficients, we can get the corresponding clusters of operators for different connectives. The operators can change continuously, and the ones gotten with the same correlation coefficient are corresponding with one another.

As long as the values of $h$ and $k$ are given, the corresponding operators of connectives can be gotten. So, in practical application, we can get the appropriate operators of connectives by the inherent correlation between propositions, this avoiding effectively giving them at will and blindly.

2. First-order universal implication

In Universal Logic, first-order universal implication is defined as: Definition $1^{[12]}$ First-order universal implication is the cluster $I(x, y, h, k)=$ ite $\left\{1|x \leq y ; 0| m \leq 0, y=0, x \neq 0 ; \Gamma^{l}\left[\left(1-x^{m n}+y^{m n}\right)^{1 /(m n)}\right]\right\}$. 
where $m=(3-4 h) /(4 h(1-h)), h \in[0,1], m \in R, n=-1 / \log _{2} k, k \in[0,1]$, $n \in R^{+}$.

Remark: the conditional expression ite $\{\beta \mid \alpha ; \gamma\}$ represents that if $\alpha$ is true, then the result is $\beta$, otherwise $\gamma$. Similarly, ite $\{\beta 1|\alpha 1 ; \beta 2| \alpha 2 ; \gamma\}=i$ te $\{\beta 1 \mid \alpha 1$; ite $\{\beta 2 \mid \alpha 2 ; \gamma\}\}$. And $\Gamma^{1}[x]=$ ite $\{1|x>1 ; 0| x<0$ or $x$ is an imaginary number; $x\}$.

For the sake of convenience, the following piecewise form is given ${ }^{[16]}$ :

$$
I(x, y, h, k)=\left\{\begin{array}{ll}
\text { ite }\{y \mid x=1 ; 1\} & \{0\} \times(0,1] \cup(0,0.75) \times\{1\} \\
\min (1, y / x) & {[0,1] \times\{0\} \cup\{0.75\} \times(0,1]} \\
\text { ite }\{1 \mid x \leq y ; y\} & (0.75,1] \times\{1\} \cup\{1\} \times(0,1) \\
1 & x \leq y,(0,0.75) \times(0,1) \cup(0.75,1) \times(0,1) \\
0 & x>y, y=0,(0.75,1) \times(0,1) \\
\left(1-x^{m+\prime}+y^{m m}\right)^{1 /(m m)} & \text { else }
\end{array}\right\}
$$

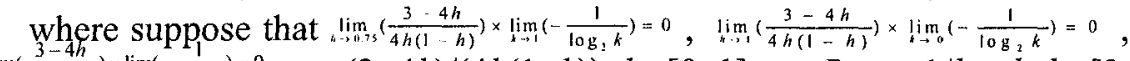
$\lim _{h \rightarrow \rightarrow \infty}\left(\frac{3-4 h}{4 h(1-h)}\right) \times \lim _{k \rightarrow 0}\left(-\frac{1}{\log _{2} k}\right)=0, m=(3-4 h) /(4 h(1-h)), h \in[0,1], m \in R, n=-1 / \log _{2} k, k \in[0$, 1], $n \in R^{+},\{0\} \times[0,1]$ represents $h=0$ and $k \in[0,1]$, and the others are similar.

The first-order universal implication is a continuous super-cluster of implication operators determined by $h$ and $k$. In practical application, according to the general correlation between propositions and the general self-correlation, we can take the corresponding one from the cluster.

3. Some common properties of first-order universal implication

For the sake of convenience, some common properties of first-order universal implication are given ${ }^{[12,16]}$ :

I1 If $x_{1} \leq x_{2}$, then $I\left(x_{1}, y, h, k\right) \geq I\left(x_{2}, y, h, k\right)$, where $h \in[0,1], k \in[0,1]$.

I2 If $y_{I} \leq y_{2}$, then $I\left(x, y_{I}, h, k\right) \leq I\left(x, y_{2}, h, k\right)$, where $h \in[0,1], k \in[0,1]$.

I3 $I(0, y, h, k)=1$, where $h \in[0,1], k \in[0,1]$.

I4 $I(1, y, h, k)=y$, where $h \in[0,1], k \in[0,1]$.

\subsection{Information boundedness principle}

Most of the discussions about the selection of the implication operator have been based on satisfaction to a number of properties associated with the classical binary implication operator ${ }^{[5,8]}$, such as the famous D-P conditions $^{[6]}$. However, these properties except continuity are all local or pointwise requirements. As said above, in considering the appropriateness of the implication operator used in FMP, account must be taken of the whole resulting fuzzy subset, not only some points in its domain ${ }^{[7-9]}$. Therefore, Yager proposed the information boundedness principle, which requires that the information contained in a fuzzy granule resulting from an inference must be no greater than the information contained in the consequent of the ifthen proposition and that the information contained in inferences under two different inputs should be ordered by the matching degree between the input and the antecedent of the if-then proposition ${ }^{[8]}$. In fact, if the implication 
operator satisfies the property $\mathrm{I} 4$, the latter contains the former. So, in the following sections, we will emphasize to discuss the latter.

In [13], Yager proposed a characterization of measure of specificity, which provides an appropriate measure of the information contained in a proposition.

Definition $2^{[13]}$ Suppose that $X$ is a finite set of cardinality $n$ and $A$ is a fuzzy subset in X. A measure $S p: F(X) \rightarrow[0,1]$ is called a measure of specificity if it satisfies the following properties:

1) $\operatorname{Sp}(A)=1$ if and only if $A$ is a singleton set, $A=\{x\}$.

2) $\operatorname{Sp}(\varnothing)=0$.

3) If $B$ and $C$ are normal fuzzy sets in $X$ and $B \subset C$, then $S p(B) \geq S p(C)$.

Of course, in practical application, we can define many different concrete models of measure of specificity. In this paper, we will use a class of specificity measures, called as linear specificity measure ${ }^{[15]}$ :

Definition $3^{[15]}$ Suppose that $X$ is a finite set of cardinality $n, A$ is a fuzzy subset in $X$ and $a_{j}$ is the $j$ th largest membership grade in $A$. A linear specificity measure is defined as

$\operatorname{Sp}(A)=a_{1}-\sum_{j=2}^{n} w_{j} a_{j}$

where and the $w_{j}$ 's are a set of weights satisfying: 1) $w_{j} \in[0,1]$; 2) $\sum_{j=2}^{\infty} w_{j}=1$; 3) $w_{i} \geq w_{j}$ for $i<j$.

\section{INFORMATION REQUIREMENT OF FIRST- ORDER UNIVERSAL IMPLICATION IN FUZZY REASONING}

\subsection{Information requirement of implication operators}

Without loss of generality, we assume that $Y$ is a finite set of cardinality $n$ : $\left\{y_{1}, y_{2}, \ldots, y_{n}\right\}$ and its elements have been indexed such that $\mu_{B}\left(y_{i}\right) \geq \mu_{B}\left(y_{j}\right)$ for $i<j$ in Eq.(1). And suppose that all propositions have the same level of measuring errors, namely the equal values for $k$ in an application. In fuzzy control, the input $x$ corresponding to the state variable is crisp, $x=x^{*}$, then $A^{\prime}$ is a singleton, that is, $\mu_{A}(x)=1$ if $x=x^{*}$, and $\mu_{A}(x)=0$ if $x \neq x^{*}$. Thus, we will get the following expression using the CRI method:

$$
\begin{aligned}
& \mu_{B^{*}}(y)=\sup T_{h_{1}, k}\left(\mu_{A^{*}}(x), I_{h_{2}, k}\left(\mu_{A}(x), \mu_{B}(y)\right)\right) \\
= & I_{h_{2}, k}\left(\mu_{A}\left(x^{*}\right), \mu_{B}(y)\right)
\end{aligned}
$$

where $T_{h_{1}, k}$ and $I_{h_{2}, k}$ are the first-order universal conjunction operator and the first-order universal implication operator determined by the $h_{1}, h_{2}$ and $k$ between propositions, respectively. 
According to the reasoning process in [17], we can similarly get the following conclusions:

Proposition 1 In fuzzy reasoning, the implication operators satisfying the information boundedness principle should satisfy the requirement, that is, the truer the consequent of the if-then proposition is, the more insensitive $I_{h, k}(x$, $y$ ) is to the change of $x$.

Proposition 2 In fuzzy reasoning, the implication operators satisfying the information boundedness principle should satisfy the requirement, that is, $D(v)=I_{h, k}(v, a)-I_{h, k}(v, b)$ is a non-decreasing function of $v$ for all $a>b$.

Obviously, Prop. 1 and Prop. 2 are equal, which describe the different aspects of one thing.

\subsection{Information requirement of first-order universal implication}

In this section, we will analyze the information requirement of the firstorder universal implication in detail, and give the general conclusion. According to its piecewise definition, we have:

1. Suppose that $m \rightarrow-\infty, n \in(0,+\infty)$, that is, $h=1, k \in(0,1)$. So $I_{h, k}(x, y)$ is Gödel implication operator $R_{G}(x, y)=i$ ite $\{1 \mid x \leq y ; y\}$.

$D(v)=I_{h, k}(v, a)-I_{h, k}(v, b)=i t e\{1 \mid v \leq a ; a\}-$ ite $\{1 \mid v \leq b ; b\}$.

1) If $v \leq b<a$, then $D(v)=I_{h, k}(v, a)-I_{h, k}(v, b)=1-1=0$.

2) If $b<v \leq a$, then $D(v)=I_{h, k}(v, a)-I_{h, k}(v, b)=1-b \geq 0$.

3) If $b<a<v$, then $D(v)=I_{h, k}(v, a)-I_{h, k}(v, b)=a-b \leq 1-b$.

According to the above analyses, $I_{h, k}(x, y)$ do not satisfy the principle.

2. Suppose that $m \in(-\infty, 0), n \rightarrow+\infty$, that is $h \in(0.75,1], k=1$. So $I_{h, k}(x, y)$ is Gödel implication operator $R_{G}$. According to the above analyses, $I_{h, k}(x, y)$ do not satisfy the principle.

3. Suppose that $m=0, n \in(0,+\infty)$, namely, $h=0.75, k \in(0,1]$. So $I_{h, k}(x, y)$ is Goguen implication operator $R_{G o}(x, y)=i t e\{1 \mid x=0 ; \min (1, y / x)\}$.

$D(v)=I_{h, k}(v, a)-I_{h, k}(v, b)=$ ite $\{1 \mid v=0 ; \min (1, a / v)\}-$ ite $\{1 \mid v=0 ; \min (1, b / v)\}$

If $v=0$, then $D(v)=I_{h, k}(v, a)-I_{h, k}(v, b)=1-1=0$.

2) If $0<v \leq b<a$, then $D(v)=I_{h, k}(v, a)-I_{h, k}(v, b)=1-1=0$.

3) If $b<v \leq a$, that is, $a / v \geq 1>b / v$, then $D(v)=1-b / v>0$, and $1-b / v$ is a nondecreasing function of $v$.

4) If $b<a<v$, that is, $1>a / v>b / v$, then $D(v)=a / v-b / v=(a-b) / v$, and $(a-b) / v$ is a non-increasing function of $v$.

Due to the above analyses, $I_{h, k}(x, y)$ do not satisfy the principle.

4. Suppose that $m \in(-\infty,+\infty), n \rightarrow 0$, namely, $h \in[0,1], k=0$. So $I_{h, k}(x, y)$ is Goguen implication operator $R_{G o}$. Due to the above analyses, $I_{h, k}(x, y)$ do not satisfy the principle. 
5. Suppose that $m \in(0,+\infty), n \rightarrow+\infty$, that is, $h \in(0,0.75), k=1$. So $I_{h, k}(x, y)$ is a variant of the Standard Sharp implication $R(x, y)=$ ite $\{y \mid x=1 ; 1\}$.

$D(v)=I_{h, k}(v, a)-I_{h, k}(v, b)=$ ite $\{a \mid v=1 ; 1\}-$ ite $\{b \mid v=1 ; 1\}$.

1) If $v \neq 1$, then $D(v)=I_{h, k}(v, a)-I_{h, k}(v, b)=1-1=0$.

2) If $\mathrm{v}=1$, then $D(v)=I_{h, k}(v, a)-I_{h, k}(v, b)=a-b>0$.

Based on the above analyses, $I_{h, k}(x, y)$ satisfy the principle.

6. Suppose that $m \rightarrow+\infty, n \in(0,+\infty)$, that is, $h=0, k \in(0,1]$. So $I_{h, k}(x, y)$ is a variant of the Standard Sharp implication. Based on the above analyses, $I_{h, k}(x, y)$ satisfy the principle.

7. Suppose that $m \in(-\infty, 0), n \in(0,+\infty)$, namely, $h \in(0.75,1), k \in(0,1)$. According to Def. 1, we have

1) If $v \leq b<a$, then $D(v)=I_{h, k}(v, a)-I_{h, k}(v, b)=1-1=0$.

2) If $b=0$, then $v>0$ and $a>0$.

i) If $v \leq a$, then $D(v)=I_{h, k}(v, a)-I_{h, k}(v, b)=1-0=1$.

ii) If $v>a$, then $D(v)=\Gamma^{1}\left[\left(1-v^{m n}+a^{m n}\right)^{1 /(m n)}\right]-0=\Gamma^{1}\left[\left(1-v^{m n}+a^{m n}\right)^{1 /(m n)}\right]$. Due to the property $\mathrm{I} 1, D(v)$ is non-increasing.

So, if $h \in(0.75,1)$ and $k \in(0,1)$, then the first-order universal implication operators do not satisfy the principle.

8. Suppose that $m \in(0,+\infty), n \in(0,+\infty)$, that is, $h \in(0,0.75), k \in(0,1)$. According to Def. 1 , we have

1) If $v \leq b<a$, then $D(v)=I_{h, k}(v, a)-I_{h, k}(v, b)=1-1=0$.

2) If $b<v \leq a$, then $D(v)=1-\Gamma^{1}\left[\left(1-v^{m n}+b^{m n}\right)^{1 /(m n)}\right] \geq 0$. Due to the property I1, $D(v)$ is a non-decreasing function of $v$, which take its maximum at $v=a$ :

$D_{1}(v)=1-\left(1-a^{m n}+b^{m n}\right)^{1 /(m n)}$

3) If $b<a<v$, then $b^{m n}<a^{m n}<v^{m n}$ and $b^{m n}, a^{m n}, v^{m n} \in[0,1]$. So we have

$I_{h, k}(v, a)=\Gamma^{1}\left[\left(1-v^{m n}+a^{m n}\right)^{1 /(m n)}\right]=\left(1-v^{m n}+a^{m n}\right)^{1 /(m n)}$

$I_{h, k}(v, b)=\Gamma^{1}\left[\left(1-v^{m n}+b^{m n}\right)^{1 /(m n)}\right]=\left(1-v^{m n}+b^{m n}\right)^{1 /(m n)}$

According to $E q .(7)$ and $E q .(8)$, we have:

$D(v)=I_{h, k}(v, a)-I_{h, k}(v, b)=\left(1-v^{m n}+a^{m n}\right)^{1 /(m n)}-\left(1-v^{m n}+b^{m n}\right)^{1 /(m n)}$

that is,

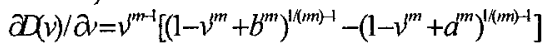

) Suppose that $n \in(1,+\infty)$, that is, $1 / n \in(0,1), k \in(0.5,1)$, fuzzy propositions have positive measuring errors for their membership grades, and we have

) $m \in[1,+\infty)$, that is, $h \in(0,0.5]$,propositions are restraining correlative.

So, $m n>1$, that is, $1 /(m n)-1<0$, and we have $\left(1-v^{m n}+b^{m n}\right)^{1 /(m n)-1}>\left(1-v^{m n}+a^{m n}\right)^{1 /(m n)-1}$, namely, $\partial D(v) / \partial v>0$. Thus, $D(v)$ is a non-decreasing function of $v$, which take its minimum at $v=a$ :

$D_{2}(v)=\left(1-a^{m n}+a^{m n}\right)^{1 /(m n)}-\left(1-a^{m n}+b^{m n}\right)^{1 /(m n)}=1-\left(1-a^{m n}+b^{m n}\right)^{1 /(m n)}$

According to $E q .(6)$ and $E q .(11)$, we have $D_{1}(v)=D_{2}(v)$. 
Thus, if $k \in(0.5,1), h \in(0,0.5]$, that is, propositions have positive measuring errors for their membership grades and are restraining correlative, then the first-order universal implication operators satisfy the principle.

) $m \in(1 / n, 1)$, namely, propositions are rejecting correlative with $h \in(0.5$, $\left.\left((n+1)-\left(n^{2}-n+1\right)^{1 / 2}\right) / 2\right)$.

So, $m n>1$, that is, $1 /(m n)-1<0$. Similarly, $D(v)$ is a non-decreasing function of $v$, which take its minimum at $v=a$, and $D_{1}(v)=D_{2}(v)$.

Thus, if propositions have positive measuring errors and are rejecting correlative with $h \in\left(0.5, \quad\left((n+1)-\left(n^{2}-n+1\right)^{1 / 2}\right) / 2\right)$, then the first-order universal implication operators satisfy the principle.

) $m=1 / n$, that is, $m n=1$, and propositions are rejecting correlative with $h=\left((n+1)-\left(n^{2}-n+1\right)^{1 / 2}\right) / 2$.

So, we have

$$
\begin{aligned}
& D(v)=I_{h, k}(v, a)-I_{h, k}(v, b)=\Gamma^{1}\left[\left(1-v^{m n}+a^{m n}\right)^{1 /(m n)}\right]-\Gamma^{1}\left[\left(1-v^{m n}+b^{m n}\right)^{1 /(m n)}\right] \\
&=(1-v+a)-(1-v+b)=a-b \\
& \text { and } D_{1}(v)=1-\left(1-a^{m n}+b^{m n}\right)^{1 /(m n)}=1-(1-a+b)=a-b .
\end{aligned}
$$

Thus, if propositions have positive measuring errors and are rejecting correlative with $h=\left((n+1)-\left(n^{2}-n+1\right)^{1 / 2}\right) / 2$ then the first-order universal implication operators satisfy the principle.

) $m \in(0,1 / n)$, that is, propositions are rejecting correlative with $h \in\left(\left((n+1)-\left(n^{2}-n+1\right)^{1 / 2}\right) / 2,0.75\right)$.

So, $\quad m n>1$, that is, $1 /(m n)-1>0$ and we have $\left(1-v^{m n}+b^{m n}\right)^{1 /(m n)-1}<\left(1-v^{m n}+a^{m n}\right)^{1 /(m n)-1}$, namely, $\partial D(v) / \partial v<0$. Thus, $D(v)$ is a decreasing function of $v$.

Thus, if propositions have positive measuring errors and are rejecting correlative with $h \in\left(\left((n+1)-\left(n^{2}-n+1\right)^{1 / 2}\right) / 2,0.75\right)$, then the first-order universal implication operators do not satisfy the principle.

) Suppose that $n \in(0,1)$, that is, $1 / n \in(1,+\infty), k \in(0,0.5)$, fuzzy propositions have negative measuring errors for their membership grades, and we have

) $m \in(1 / n,+\infty)$, that is, propositions are restraining correlative with $h \in(0$, $\left.\left((n+1)-\left(n^{2}-n+1\right)^{1 / 2}\right) / 2\right)$.

So, $m n>1$, that is, $1 /(m n)-1<0$. Similarly, $D(v)$ is a non-decreasing function of $v$, which take its minimum at $v=a$, and $D_{1}(v)=D_{2}(v)$.

Thus, if propositions have negative measuring errors and are restraining correlative with $h \in\left(0,\left((n+1)-\left(n^{2}-n+1\right)^{1 / 2}\right) / 2\right)$, then the first-order universal implication operators satisfy the principle.

) $m=1 / n$, that is, $m n=1$, and propositions are restraining correlative with $h=\left((n+1)-\left(n^{2}-n+1\right) 1 / 2\right) / 2$.

Similarly, we have $D(v)=a-b=D_{1}(v)$. 
) $m=1 / n$, that is, $m n=1$, and propositions are restraining correlative with $h=\left((n+1)-\left(n^{2}-n+1\right) 1 / 2\right) / 2$.

Similarly, we have $D(v)=a-b=D_{1}(v)$.

Thus, if propositions have negative measuring errors and are restraining correlative with $h=\left((n+1)-\left(n^{2}-n+1\right)^{1 / 2}\right) / 2$, then the first-order universal implication operators satisfy the principle.

) $m \in[1,1 / n)$, that is, that is, propositions are restraining correlative with $h \in\left(\left((n+1)-\left(n^{2}-n+1\right)^{1 / 2}\right) / 2,0.5\right]$.

So, $m n<1$, that is, $1 /(m n)-1>0$. Similarly, $D(v)$ is a decreasing function of $v$.

Thus, if propositions have negative measuring errors and are restraining correlative with $h \in\left(\left((n+1)-\left(n^{2}-n+1\right)^{1 / 2}\right) / 2,0.5\right]$, then the first-order universal implication operators do not satisfy the principle.

) $m \in(0,1)$, that is, $h \in(0.5,0.75)$, and propositions are rejecting correlative.

So, $m n<1$, that is, $1 /(m n)-1>0$. Similarly, $D(v)$ is a decreasing function of $v$.

Thus, propositions have negative measuring errors and are rejecting correlative, then the operators do not satisfy the principle.

) Suppose that $n=1$, that is, $k=0.5$, fuzzy propositions have no measuring errors for their membership grades. Then first-order universal implication changes into zero-order universal implication.

So, we have the conclusion that when propositions are restraining correlative, that is, $h \in[0,0.5]$, the universal implication operators satisfy the information boundedness principle ${ }^{[17]}$.

Fig.1-2 shows $I_{h, k}(v, a), I_{h, k}(v, b)$ and $D(v)$ for several representative values of $h$, where $a=0.6, b=0.1$, with $k=0.7$ or 0.125 , respectively.
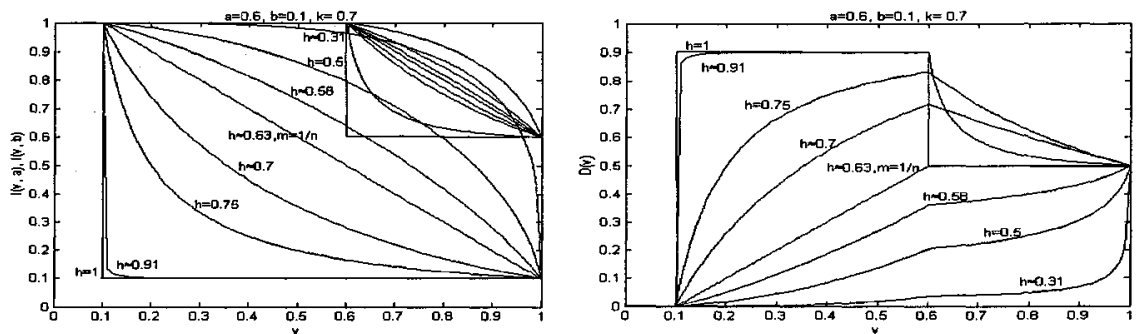

Fig. 1 When measuring errors are positive, $J(v, a), I(v, b)$ and $D(v)$ for several different values of $h$
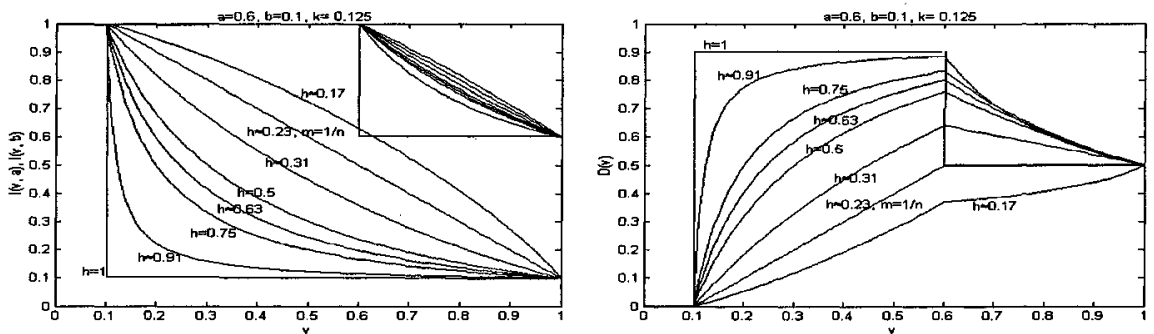

Fig. 2 When measuring errors are negative, $I(v, a), I(v, b)$ and $D(v)$ for several different values of $h$ 
Based on the above analyses about information requirement of the firstorder universal implication operators, we can draw the conclusion as follows:

When propositions are attracting correlative, namely, $h \in[0.75,1]$, the first-order universal implication operators do not satisfy the information boundedness principle with propositions having positive or negative measuring errors;

When propositions have positive measuring errors, if they are rejecting correlative with $h \in\left(\left((n+1)-\left(n^{2}-n+1\right)^{1 / 2}\right) / 2,0.75\right)$, then the first-order universal implication operators do not satisfy the principle; and if they are rejecting correlative with $h \in\left[0.5,\left((n+1)-\left(n^{2}-n+1\right)^{1 / 2}\right) / 2\right]$ and restraining correlative with $h \in[0,0.5]$, then the operators satisfy the principle;

When propositions have negative ones, if they are rejecting correlative with $h \in(0.5,0.75]$ and restraining correlative with $h \in\left(\left((n+1)-\left(n^{2}-n+1\right)^{1 / 2}\right) / 2,0.5\right]$, then the first-order universal implication operators do not satisfy the principle; and if they are restraining correlative with $h \in\left[0,\left((n+1)-\left(n^{2}-n+1\right)^{1 / 2}\right) / 2\right]$, then the operators satisfy the principle;

When propositions have positive maximal measuring error, namely $k=1$, if they are rejecting or restraining correlative, then the first-order universal implication operators satisfy the principle; and when they have negative maximal one, namely $k=0$, all operators do not satisfy the principle.

\section{CONCLUSIONS}

Based on the definition of linear specificity measure, this paper has discussed detailedly the conditions on which the first-order universal implication operators satisfy the information boundedness principle in fuzzy reasoning, and get the conclusion: When propositions have positive measuring errors, if they are rejecting correlative with $h \in[0.5$, $\left.\left((n+1)-\left(n^{2}-n+1\right)^{1 / 2}\right) / 2\right]$ or restraining correlative with $h \in[0,0.5]$, then the first-order universal implication operators satisfy the principle; and when propositions have negative ones, if they are restraining correlative with $h \in[0$, $\left.\left((n+1)-\left(n^{2}-n+1\right)^{1 / 2}\right) / 2\right]$, then the first-order universal implication operators satisfy the principle. This conclusion has important directive meaning for how to give the value of the general correlative coefficient $h$ in practical control application.

As far as other models or a general expression for families of measure of specificity based on t-norms of a fuzzy set ${ }^{[14]}$, what is the information requirement of the first-order universal implication operators? Is there some restraining relation between the t-norms and the t-norms used to generate the 
corresponding first-order universal implication operators? These problems will be further studied elsewhere.

\section{ACKNOWLEDGEMENTS}

This work was supported by the Foundation of Natural Sciences under Grant 60273087, the National Plan of 863 under Grant 2002AA412020 and the Foundation of Natural Sciences of Beijing under Grant 4032009.

\section{REFERENCES}

1. Mizumoto M, Zimmermann H. Comparison of Fuzzy Reasoning Method. Fuzzy Sets and Systems, 1982, 8:253-283

2. Fernandez F. G., Kreinovich V. Fuzzy Implication Can be Arbitrarily Complicated: A Theorem. Int. J. Intelligent Systems, 1998, 13:445-451

3. Turksen I. B., Kreinovich V., Yager R. R., A new class of fuzzy implications. Axioms of fuzzy implication revisited. Fuzzy Sets and Systems, 1998, 100:262-272

4. Dujet C., Vincent N. Force implication:A new approach to human reasoning. Fuzzy Sets and Systems, 1995, 69:53-63

5. Klir G. J., Bo Y. Fuzzy Sets and Fuzzy Logic: Theory and Application, Prentice-Hall, NJ, 1995

6. D. Dubois, H. Parde. Fuzzy sets in approximate reasoning part I: inference with possibility distributions, Fuzzy Sets and Systems, 1991, 40:143-202

7. Yager R. R. Measures of information in generalized constraints. Int. J. uncertainty, fuzziness and knowledge-based systems, 1998, 6:519-532

8. Yager R. R. On global requirements for implication operators in fuzzy modus ponens. Fuzzy S. and S., 1999,106:3-10

9. D.Chen, Information measure requirement of implication operator in fuzzy reasoning,Comp. Sci.,2001,28(8):124-126.

10. H.C. He, Generalized logic in experience thinking, Science in China(Series E), 1996, 39(3):225-234.

11. H.C. He, Studies on generalized implication operation and generalized series reasoning operation, Journal of Software, 1998, 9(6):469-473(in Chinese).

12. H.C. He, Universal logics principle. Science Press, Beijing, 2001 (in Chinese).

13. R. R. Yager, On the specificity of a possibility distribution, Fuzzy Sets and Systems, 1992, 50:279-292.

14. L. Garmendia, On t-norms based measures of specificity, Fuzzy Sets and Systems, 2003, 133:237-248.

15. R. R. Yager, Default knowledge and measures of specificity, Inform. Sci. 1992, 61:1-44.

16. Y.C. Ma, The properties of universal implication, to appear(in Chinese).

17. L.H. Fu, Research on information requirement of zero-order universal implication operators in fuzzy reasoning, The $5^{\text {th }}$ WCICA, (accepted) 